\title{
Anti-IL-5 Agents for the Treatment of Idiopathic Chronic Eosinophilic Pneumonia: A Case Series
}

\author{
Hiroki Tashiro, Koichiro Takahashi (D), Yuki Kurihara, Hironori Sadamatsu, Yuki Kuwahara, \\ Shinya Kimura, Naoko Sueoka-Aragane \\ Division of Hematology, Respiratory Medicine and Oncology, Department of Internal Medicine, Faculty of Medicine, Saga University, Saga, Japan
}

Correspondence: Hiroki Tashiro, Division of Hematology, Respiratory Medicine and Oncology, Department of Internal Medicine, Faculty of Medicine, Saga University, 5-I-I Nabeshima, Saga, Saga Prefecture, 849-850I, Japan, Tel +8I-952-34-2369, Fax +8I-952-34-20I7, Email si3222@cc.saga-u.ac.jp

Purpose: Idiopathic chronic eosinophilic pneumonia (ICEP) is a rare, chronic respiratory disease. Corticosteroid therapy is effective for ICEP, but relapse is frequent after its tapering, which leads to chronic use and corticosteroid-related adverse effects. Currently, biological agents targeting interleukin 5 (IL-5) are considered alternatives for treating ICEP patients with frequent relapse, but the detailed effects are not fully understood.

Patients and Methods: The clinical characteristics of 30 patients with ICEP, especially 12 patients with ICEP who experienced relapse after corticosteroid dose tapering, were evaluated retrospectively. In addition, 4 ICEP patients with frequent relapse treated by IL-5-targeted biological agents were reviewed.

Results: Of the 30 patients diagnosed with ICEP, 12 patients $(40.0 \%)$ recurred after corticosteroid dose tapering, and 9 (30.0\%) were treated with maintenance doses of corticosteroid. Of ICEP patients who experienced recurrence, $6(50.0 \%)$ had frequent relapses $(2$ or more times). All 4 patients treated with anti-IL-5 agents had their corticosteroid dose reduced without any relapses; in 3 patients, corticosteroids were withdrawn.

Conclusion: Anti-IL-5 agents might be alternatives for treating ICEP patients with frequent relapses.

Keywords: chronic eosinophilic pneumonia, idiopathic, anti-IL-5 agents, mepolizumab, benralizumab

\section{Introduction}

Chronic eosinophilic pneumonia (CEP) is a rare, chronic respiratory disease that is characterized by infiltration of eosinophils in lung parenchyma. ${ }^{1,2}$ There are various causes of CEP, including: drugs, mycosis, parasitic infestation, and other systemic eosinophil-activated diseases such as eosinophilic granulomatosis with polyangiitis, allergic bronchopulmonary mycosis, ${ }^{3,4}$ and idiopathic chronic eosinophilic pneumonia (ICEP). Patients with ICEP have prolonged fever, cough, and sputum, and they show multiple pulmonary infiltrates on chest radiography. ${ }^{1,5,6}$ Blood eosinophilia is definitely seen, with levels of more than $6 \%$ of the total white blood cells or $1000 / \mu \mathrm{L}{ }^{5}$ In addition, bronchoscopy plays a role in the diagnosis of CEP, since bronchoalveolar lavage fluid (BALF) shows more than $25 \%$ eosinophils. ${ }^{1}$ Most patients with CEP recover after corticosteroid treatment. ${ }^{7}$ However, relapse is frequent after tapering of the corticosteroid dose,${ }^{8-10}$ which leads to long-term corticosteroid administration, resulting in corticosteroid-related adverse effects such as diabetes mellitus, infections, osteoporosis, and glaucoma, ${ }^{11}$ which are important clinical issues in the management of ICEP.

Recently, interleukin 5 (IL-5)-targeted biological agents such as mepolizumab and benralizumab have been used for the treatment of severe eosinophilic asthma. ${ }^{12,13}$ IL-5 is a major cytokine that is related to eosinophil differentiation, proliferation, activation, and eosinophil-mediated inflammatory responses. ${ }^{14}$ Mepolizumab is a humanized monoclonal antibody that affects the IL-5 $\alpha$-chain and prevents its binding to the $\alpha$ subunit of the IL-5 receptor. ${ }^{15}$ On the other hand, benralizumab binds to the IL-5R $\alpha$ subunit expressed on eosinophils, enhancing antibody-dependent cellular cytotoxicity and reducing circulating levels of eosinophils. ${ }^{16}$ Importantly, these anti-IL-5 agents have been in focus as alternative 
treatments for ICEP patients with frequent relapses on corticosteroid treatment. However, the detailed effects of IL5-targeted biological agents for ICEP have not been fully assessed.

In the present study, the clinical characteristics of 30 patients with ICEP were evaluated, with a special focus on the features of 12 patients with ICEP who experienced relapses after corticosteroid dose tapering. In addition, 4 ICEP patients with frequent relapses who were treated by IL-5 targeted biological agents were reviewed. Current data for the clinical efficacy of IL-5-targeted biological agents for ICEP patients would contribute to reducing relapses and steroidrelated adverse effects.

\section{Materials and Methods}

\section{Patients and Diagnosis}

Thirty patients diagnosed with ICEP at Saga University Hospital between 2005 and 2020 were reviewed retrospectively. All patients were diagnosed with ICEP by two expert pulmonary physicians referring to the following diagnostic criteria from the previous reports, ${ }^{1,5}$ and patients who satisfied all of the criteria were diagnosed as having ICEP: 1) clinical symptoms such as fever $\left(>37.5^{\circ} \mathrm{C}\right)$, cough, and sputum lasting more than 2 weeks; 2$)$ pulmonary infiltrates on chest imaging; 3) blood eosinophilia $>1000 / \mu \mathrm{L}$ and/or eosinophilia in BALF, with eosinophils accounting for more than $25 \%$ of the total cell count; 4) exclusion of other etiologies such as drugs, mycosis, parasitic infestation, or other systemic eosinophil-activated diseases such as eosinophilic granulomatosis with polyangiitis and allergic bronchopulmonary mycosis; and 5) improvement of the abnormalities after initiation of corticosteroid treatment. The rate of corticosteroid tapering was determined by the physician. Recurrence was also diagnosed by the physician considering the symptoms, blood eosinophilia, alveolar eosinophilia, worsening on chest imaging, and recovery of the abnormalities after treatment with increasing corticosteroid doses.

This study was approved by the ethics committee of Saga University Hospital (approval number: 2021-07-R-01, approval date: September 29, 2021) and was performed in accordance with the 1964 Declaration of Helsinki. Informed consent was obtained in the form of opt-out on the website. Those who rejected were excluded. In addition, written, informed consent for publication of their case details was obtained from each of the 4 patients with ICEP treated by antiIL-5 agents.

\section{Comorbidities and Examinations}

Comorbidities including hypertension, hyperlipidemia, diabetes mellitus, and allergic comorbidities such as asthma and allergic rhinitis were identified at the time of ICEP diagnosis. All patients were examined by chest computed tomography (CT). Symptoms and examination results for the radiological findings, laboratory findings, and the eosinophil ratio of BALF were determined at the time of ICEP diagnosis. Bronchoscopy was performed considering the risk of increased hypoxia and the patient's consent. Thus, 22 patients (73.3\%) underwent bronchoscopy for the diagnosis of ICEP.

\section{Results}

\section{Clinical Characteristics and Comorbidities in Patients with ICEP}

Thirty patients satisfied the diagnostic criteria of ICEP. Their average age was 60.9 years, and 12 patients (40.0\%) were male. The average body mass index (BMI) was $21.3 \mathrm{~kg} / \mathrm{m}^{2}$, and the duration of observation was 1553.5 days. As comorbidities, 11 patients (36.6\%) had hypertension, 5 patients (16.7\%) had hyperlipidemia, and 6 patients $(20.0 \%)$ had diabetes mellitus. As allergic comorbidities, 9 patients (30.0\%) had asthma, and 5 patients (16.7\%) had allergic rhinitis. As symptoms, 13 patients (43.3\%) had fever, 26 patients (86.7\%) had cough, and 12 patients (40.0\%) had sputum. According to radiological findings, 4 patients (13.3\%) showed abnormalities limited to one lobe, and 23 patients (76.7\%) had abnormalities distributed in both lung fields on chest CT. According to laboratory findings, the average white blood cell count was $11,734.7 / \mu \mathrm{L}$, the ratio of eosinophils was $23.8 \%$, the eosinophil count was $3392.2 / \mu \mathrm{L}$, and C-reactive protein was $5.2 \mathrm{mg} / \mathrm{dL}$. Twenty-two patients (73.3\%) underwent bronchoscopy with evaluation of alveolar eosinophilia. The average eosinophil ratio in BALF was $50.9 \%$ of the total cell count. The initial corticosteroid dose converted to prednisolone was $32.0 \mathrm{mg}$, and the average duration was 12.4 days. Twelve $(40.0 \%)$ patients had recurrence of ICEP 
Table I Clinical Characteristics of Patients with Idiopathic Chronic Eosinophilic Pneumonia $(n=30)$

\begin{tabular}{|c|c|}
\hline Age (years) & $60.9 \pm 16.4$ \\
\hline Gender (male/female) & $12 / 18$ \\
\hline BMI $\left(\mathrm{kg} / \mathrm{m}^{2}\right)$ & $21.3 \pm 3.7$ \\
\hline Duration of observation (days) & $1553.5 \pm 1326.6$ \\
\hline \multicolumn{2}{|l|}{ Comorbidities (\%) } \\
\hline Hypertension & $11(36.6 \%)$ \\
\hline Hyperlipidemia & $5(16.7 \%)$ \\
\hline Diabetes & $6(20.0 \%)$ \\
\hline \multicolumn{2}{|l|}{ Allergic comorbidities (\%) } \\
\hline Asthma & $9(30.0 \%)$ \\
\hline Allergic Rhinitis & $5(16.7 \%)$ \\
\hline \multicolumn{2}{|l|}{ Symptoms (\%) } \\
\hline Fever $\left(>37.5^{\circ} \mathrm{C}\right)$ & $13(43.3 \%)$ \\
\hline Cough & $26(86.7 \%)$ \\
\hline Sputum & $12(40.0 \%)$ \\
\hline \multicolumn{2}{|l|}{ Radiologic features on chest computed tomography (\%) } \\
\hline Limited in one lobe & $4(13.3 \%)$ \\
\hline Both lung fields & $23(76.7 \%)$ \\
\hline \multicolumn{2}{|l|}{ Laboratory findings } \\
\hline White blood cell $(/ \mu \mathrm{L})$ & $11,734.7 \pm 6475.1$ \\
\hline Eosinophil (\%) & $23.8 \pm 16.3$ \\
\hline Eosinophil count $(/ \mu \mathrm{L})$ & $3392.2 \pm 5023.3$ \\
\hline$C$ reactive protein $(\mathrm{mg} / \mathrm{dl})$ & $5.2 \pm 5.8$ \\
\hline Eosinophil ratio on bronchoalveolar lavage $(n=22)(\%)$ & $50.9 \pm 19.3$ \\
\hline Initial corticosteroid dose converted to prednisolone (mg) & $32.0 \pm 14.7$ \\
\hline Duration of the initial corticosteroid dose (days) & $12.4 \pm 6.8$ \\
\hline Patients who experienced recurrence after corticosteroid tapering (\%) & $12(40.0 \%)$ \\
\hline Patients who were treated with maintenance dose of corticosteroid (\%) & $9(30.0 \%)$ \\
\hline Maintenance dose of corticosteroid converted to prednisolone $(\mathrm{mg})$ & $7.6 \pm 5.7$ \\
\hline
\end{tabular}

Abbreviation: BMI, body mass index.

more than once, and $9(30 \%)$ patients were treated by maintenance doses of corticosteroid. The maintenance dose of corticosteroid converted to prednisolone of the 9 patients was $7.6 \mathrm{mg}$ (Table 1).

\section{Clinical Data of 12 Patients with Recurrence of ICEP After Steroid Tapering}

For the 12 patients with recurrence of ICEP after steroid tapering, the average duration from diagnosis to the first recurrence was 250.7 days. The average prednisolone dose at the time of recurrence was $5.0 \mathrm{mg} /$ day. Six patients $(50.0 \%)$ had one recurrence, and 6 patients $(50.0 \%)$ had more than 2 recurrences. According to laboratory findings at the time of recurrence, the average white blood cell count was $10,764.2 / \mu \mathrm{L}$, the ratio of eosinophils was $15.6 \%$, the eosinophil count was $1988.5 / \mu \mathrm{L}$, and C-reactive protein was $1.3 \mathrm{mg} / \mathrm{dL}$ (Table 2).

\section{Clinical Course of the 4 Patients with ICEP Treated by Anti-IL-5 Agents}

Four patients with frequent ICEP recurrences and difficulties in corticosteroid dose reduction were treated by anti-IL-5 agents (Table 3). Two patients were treated with benralizumab, which is a monoclonal antibody that binds to IL-5 receptor alpha, at $30 \mathrm{mg}$ every 4 weeks for 3 doses and then once every 8 weeks. ${ }^{17}$ The other 2 patients were treated with mepolizumab, which is a monoclonal antibody that binds to IL-5 itself, at $100 \mathrm{mg}$ every 4 weeks. ${ }^{18}$

\section{Case I}

A 67-year-old woman who had asthma and diabetes mellitus presented with cough and dyspnea. She was admitted to another hospital, and her chest radiograph showed infiltrations with blood eosinophilia. She had no evidence of other potential 
Table 2 Patients with ICEP Who Experienced Recurrence After Steroid Tapering $(n=12)$

\begin{tabular}{|l|c|}
\hline Duration from diagnosis to the first recurrence (days) & $250.7 \pm 168.7$ \\
Prednisolone dose at the time of recurrence $(\mathrm{mg})$ & $5.0 \pm 4.2$ \\
Recurrence time $(\%)$ & $6(50.0 \%)$ \\
I & $6(50.0 \%)$ \\
$>$ or $=$ to 2 & \\
Laboratory findings at the time of recurrence & $10,764.2 \pm 4637.7$ \\
White blood cell $(/ \mu \mathrm{L})$ & $15.6 \pm 14.6$ \\
Eosinophil $(\%)$ & $1988.5 \pm 2474.2$ \\
Eosinophil count $(/ \mu \mathrm{L})$ & $1.3 \pm 1.4$ \\
C reactive protein $(\mathrm{mg} / \mathrm{dl})$ & \\
\hline
\end{tabular}

Abbreviation: ICEP, idiopathic chronic eosinophilic pneumonia.

Table 3 Clinical Information of 4 ICEP Cases Treated by Anti-IL-5 Agents

\begin{tabular}{|l|c|c|l|l|l|l|l|l|}
\hline & Age & Gender & $\begin{array}{l}\text { Frequency } \\
\text { of } \\
\text { Recurrence }\end{array}$ & $\begin{array}{l}\text { Blood } \\
\text { Eosinophil } \\
(\boldsymbol{\mu L})\end{array}$ & $\begin{array}{l}\text { BAL } \\
\text { Eosinophil } \\
(\%)\end{array}$ & $\begin{array}{l}\text { Prednisolone } \\
\text { Dose at } \\
\text { Recurrence } \\
(\mathbf{m g})\end{array}$ & $\begin{array}{l}\text { Anti-IL-5 } \\
\text { Agents }\end{array}$ & $\begin{array}{l}\text { Prednisolone Dose after } \\
\text { Induction of Anti-IL-5 } \\
\text { Agents (mg) }\end{array}$ \\
\hline Casel & 67 & F & 3 & 2990 & 40 & 5 & Benralizumab & 0 \\
Case2 & 37 & F & 4 & 2853.1 & 50 & 12.5 & Benralizumab \\
Case3 & 74 & M & 2 & 4939 & n.e. & Mepolizumab & 5 \\
Case4 & 68 & M & 3 & 6068.2 & n.e. & 5 & Mepolizumab & 0 \\
\hline
\end{tabular}

Abbreviations: ICEP, idiopathic chronic eosinophilic pneumonia; BAL, bronchoalveolar lavage; IL-5, interleukin 5; n.e, not examined.

etiologies of CEP, including drugs, mycosis, or other systemic eosinophil-activated diseases, which indicated ICEP. She was treated with corticosteroid, achieving temporary control. However, 540 days after treatment initiation, the disease recurred with tapering of the prednisolone to $5 \mathrm{mg}$, and she developed 3 recurrences. Therefore, she was referred to our hospital for improvement of ICEP and worsening of glucose tolerance treated by 2 kinds of oral hypoglycemic drugs and glucagon-like peptide-1 agonist. Her chest CT findings showed ground-glass shadows and infiltrations in bilateral central lung fields (Figure 1), and the blood eosinophil count was 2990/ $\mathrm{L}$, hemoglobin Alc (HbAlc) was 6.6\%, and the alveolar eosinophil ratio was $40 \%$ in BALF. She was treated with benralizumab considering her increased risk of steroid-related adverse events. Her forced expiratory volume in 1 second recovered from $1.00 \mathrm{~L}$ to $2.03 \mathrm{~L}$ on pulmonary function testing, and the blood eosinophil count was reduced to 0 after 4 weeks. She has currently been on treatment with benralizumab for 8 months without corticosteroid, with no recurrence and well-controlled glucose levels without any diabetes drugs.

\section{Case 2}

A 37-year-old woman without comorbidities presented with fever, cough, sputum, and dyspnea for 3 weeks and was referred to our hospital. She had no hypoxia, and her chest CT showed infiltrations in bilateral peripheral lung fields (Figure 2). Her blood eosinophil count was $2853.1 / \mu \mathrm{L}$, and the alveolar eosinophil ratio was $50 \%$ in BALF. She had no other etiologies for CEP; therefore, ICEP was diagnosed. She was treated with corticosteroid and developed recurrence 182 days after treatment initiation with tapering her prednisolone to $12.5 \mathrm{mg}$. She had also experienced 4 recurrences. Considering her future risk of steroid-related adverse events, anti-IL-5 agent treatment was started. Her blood eosinophil count was reduced to 0 after 4 weeks. She has currently been on treatment with benralizumab for 4 months with $5 \mathrm{mg}$ of prednisolone, and no recurrence has been seen. Reduction of the corticosteroid dose is planned. 


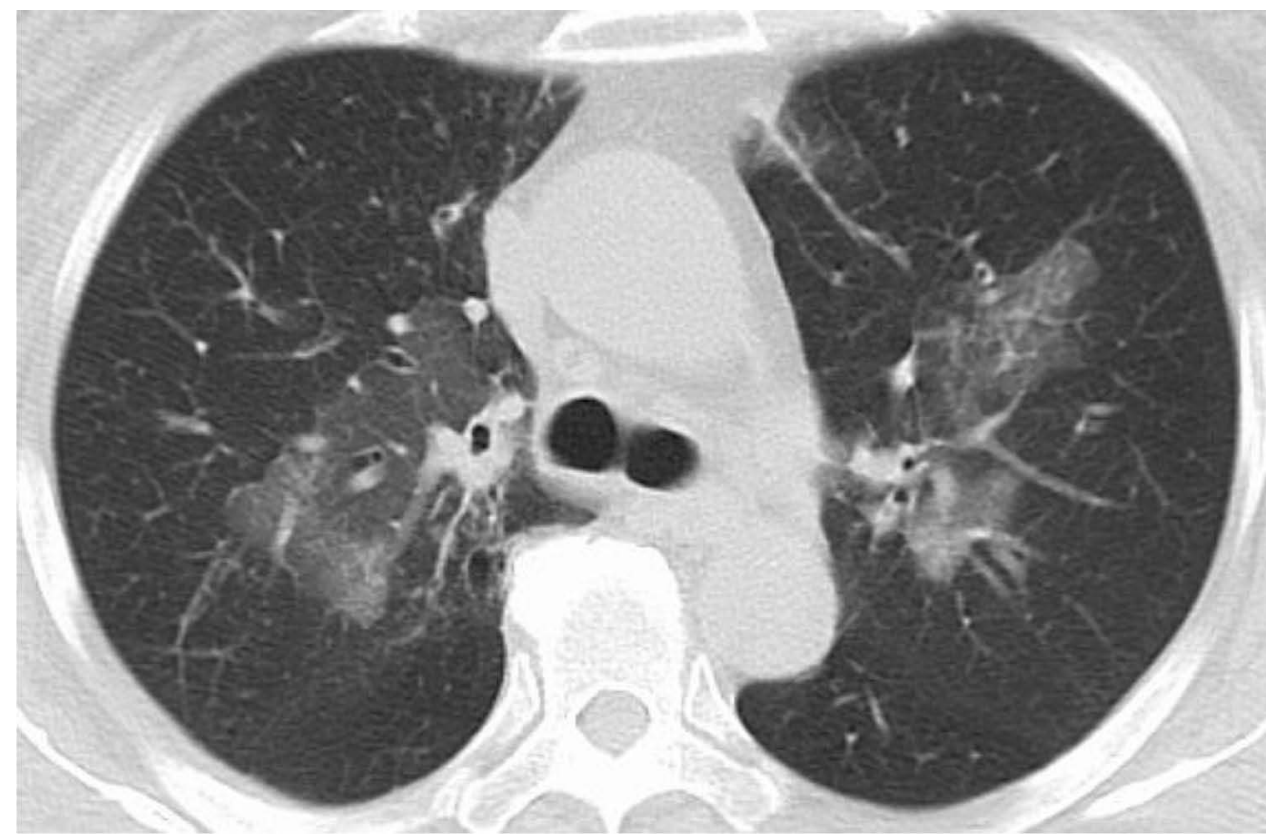

Figure I Chest computed tomography (CT) with the lung window setting shows ground-glass shadows and infiltrations in bilateral central lung fields.

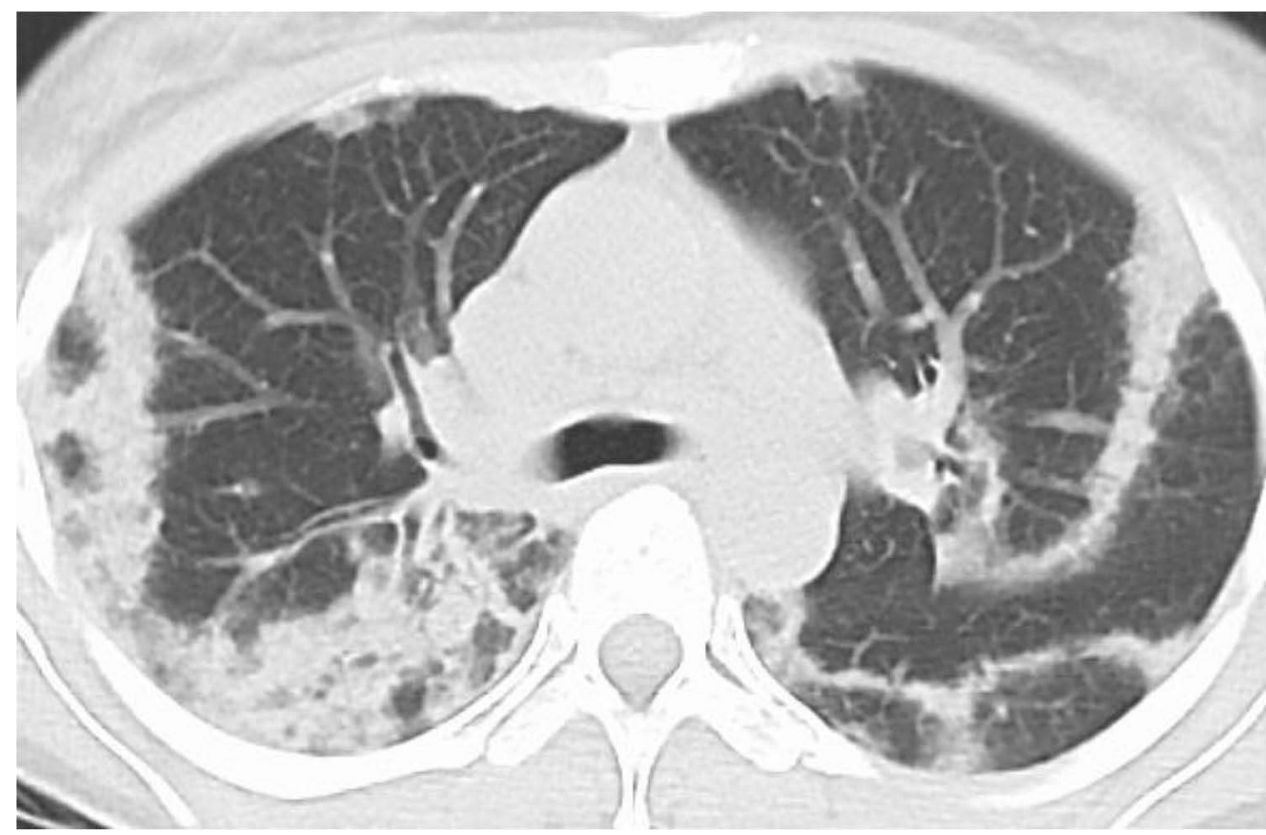

Figure 2 Chest CT with the lung window setting shows infiltrations in bilateral peripheral lung fields.

\section{Case 3}

A 74-year-old man with hypertension, hyperlipidemia, and chronic heart failure caused by an old myocardial infarction presented with cough and sputum for 2 weeks and was referred to our service. His chest CT showed bilateral infiltrations (Figure 3), and his blood eosinophil count was $4939 / \mu \mathrm{L}$. Bronchoscopy was not performed due to his unstable cardiac status. Since other causes of CEP were ruled out, ICEP was diagnosed. He was treated with corticosteroid, and his symptoms, chest CT findings, and blood eosinophilia recovered, but they re-appeared again 238 days after treatment 


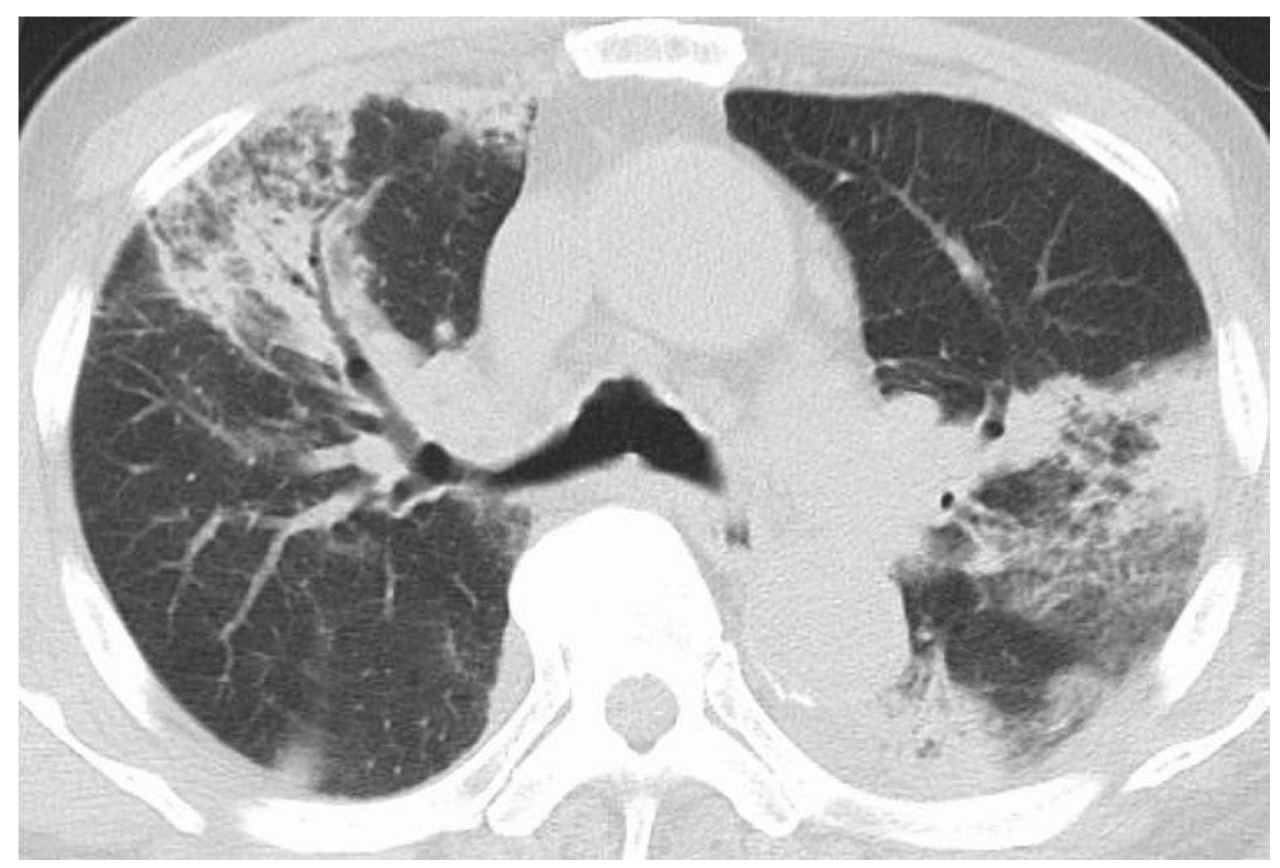

Figure 3 Chest CT with the lung window setting shows infiltrations in bilateral lung fields.

initiation with a prednisolone dose of $4 \mathrm{mg}$. He also developed 2 recurrences, and there was concern about progression of arteriosclerosis that could cause another cardiac event. He was treated with mepolizumab, and his blood eosinophil count was reduced to $32.5 / \mu \mathrm{L}$ after 4 weeks. He has currently been treated with mepolizumab for 19 months and has not experienced any recurrences without corticosteroid.

\section{Case 4}

A 68-year-old man with diabetes mellitus and cough and sputum for 2 weeks was admitted to another hospital. His chest CT showed ground-glass shadows and infiltrations in bilateral lower lung fields (Figure 4). His blood eosinophil count was $6068 / \mu \mathrm{L}$, and his $\mathrm{HbA1c}$ was $7.3 \%$. Bronchoscopy was not performed because he refused. Other causes of CEP were ruled out, so ICEP was diagnosed. He was treated with corticosteroid, but developed recurrence 128 days after treatment initiation with a tapered prednisolone dose of $5 \mathrm{mg}$. In addition, he experienced 3 recurrences and was maintained with $5 \mathrm{mg}$ of prednisolone. Considering the improvement of ICEP and the worsening glucose tolerance induced by corticosteroid, he was referred to our hospital. He was treated with mepolizumab, and his blood eosinophil count was reduced to $79 / \mu \mathrm{L}$ after 4 weeks. He has been treated with mepolizumab for 17 months, and no recurrences of ICEP have appeared without corticosteroid. His glucose tolerance also recovered, and $\mathrm{HbAlc}$ was $5.5 \%$.

\section{Discussion}

The present longitudinal study showed that recurrence of ICEP occurred frequently, which leads to prolonged corticosteroid treatment. Hence, corticosteroid-related adverse events should be taken into consideration in the management of ICEP. Four ICEP patients with frequent recurrences requiring maintenance doses of corticosteroid were treated with antiIL-5 agents. In all of them, the corticosteroid dose was decreased; in fact, in 3 of 4 cases, the corticosteroid was withdrawn, and all cases have not experienced additional recurrences of ICEP after the administration of anti-IL-5 agents despite the reduction of the corticosteroid dose. To the best of our knowledge, this is the first report of a case series of ICEP treated by anti-IL-5 agents.

Patients with ICEP normally respond well to corticosteroid treatment, ${ }^{1}$ and all of the patients in the present study also recovered from ICEP after initial corticosteroid treatment. During corticosteroid tapering or discontinuation, recurrence occurred frequently, as previously reported. Approximately $40 \%$ to $60 \%$ of patients with CEP experienced relapses, ${ }^{8-10,19}$ 


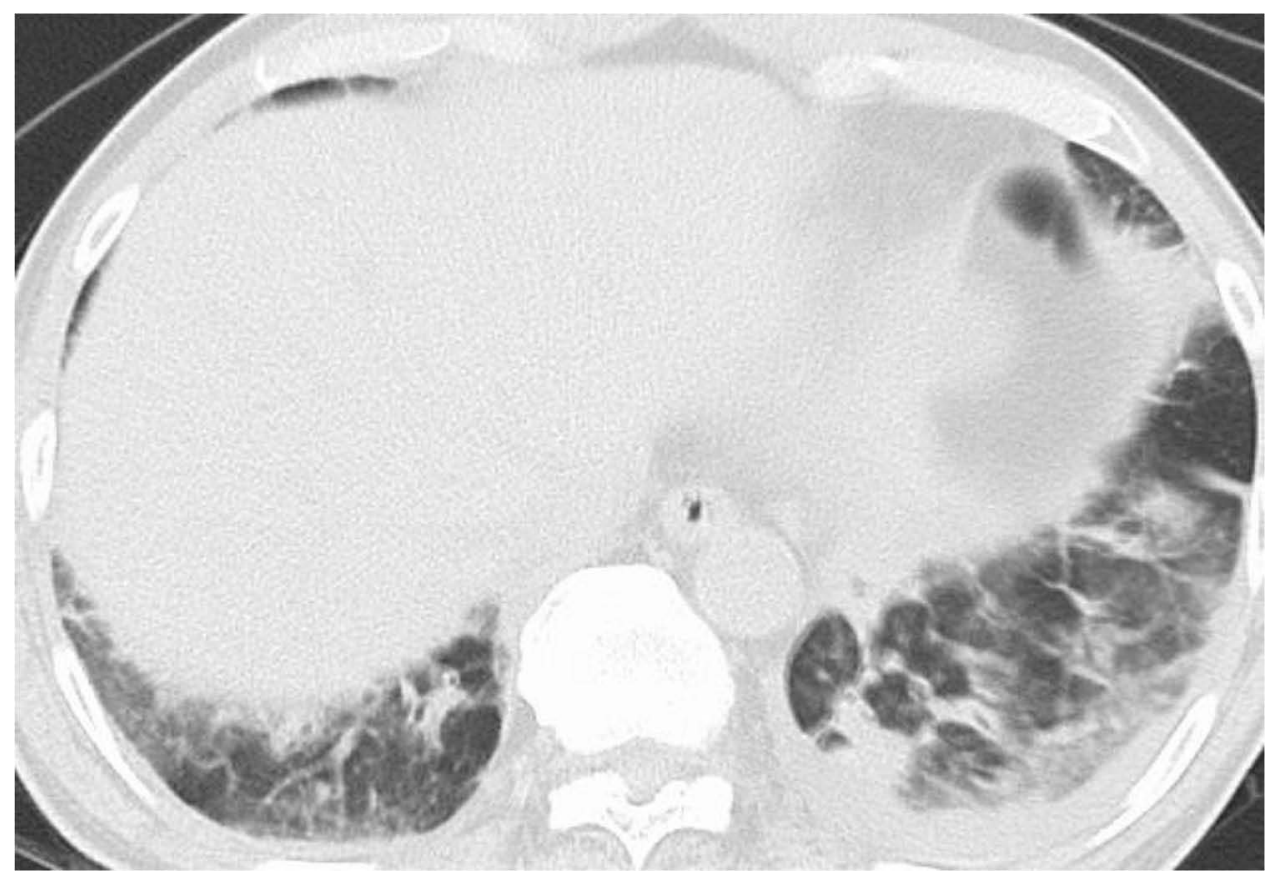

Figure 4 Chest CT with the lung window setting shows ground-glass shadows and infiltrations in bilateral lower lung fields.

and $28.6 \%$ suffered frequent relapses, 2 or more times. ${ }^{8}$ Indeed, in the present study, $40 \%$ of patients diagnosed with ICEP experienced recurrence, and 20\% relapsed 2 or more times (Tables 1 and 2) which supported the previous reports. To avoid recurrence, corticosteroid was administered as maintenance therapy, and $30 \%$ to $50 \%$ of patients were continuously treated with corticosteroid in the present study (Table 1) and previous reports, ${ }^{8,9,20}$ which results in corticosteroid-related adverse effects, such as worsening glucose tolerance, infections, osteoporosis, and glaucoma. ${ }^{11}$ Thus, alternative therapy for ICEP is necessary to reduce corticosteroid use and attenuate frequent relapses, focusing on the specific treatment target, considering efficacy and drug-induced adverse effects.

Eosinophils can be important cells in the pathophysiology of ICEP and the treatment target, because blood eosinophils and alveolar eosinophils are extremely elevated in patients with ICEP, and they are reduced after treatment, such as corticosteroid therapy. ${ }^{1}$ A previous study reported that eosinophils are detected in intra-alveolar exudate and infiltrate into interstitial sites on pathological examination. ${ }^{21}$ Notably, eosinophils induce robust inflammation at the infiltrated site with cytotoxic activity, ${ }^{22,23}$ releasing intracellular chemical contents caused by eosinophil extracellular trap cell death (EETosis). ${ }^{24}$ These data show that the mechanistic basis of ICEP involves activation and migration of eosinophils, and its specific resolution by treatment is crucial from the perspective of efficacy and reducing drug-induced adverse effects. Currently, there is increasing evidence that IL-5 is the pivotal molecule for the homeostasis of eosinophils. For example, IL-5 contributes to longer survival and activation of eosinophils, ${ }^{25}$ and IL-5 is also involved in migration of eosinophils to lung tissues. ${ }^{26,27}$ Importantly, the concentration of IL-5 in BALF of patients with CEP is significantly higher than that of healthy volunteers, ${ }^{28,29}$ which suggests that IL-5 can be a specific target as an alternative treatment for ICEP.

Currently, anti-IL-5 agents including benralizumab, mepolizumab, and reslizumab are available for patients with severe eosinophilic asthma and have the capacity to reduce blood ${ }^{12,13,30}$ and airway eosinophils. ${ }^{16,31}$ These data show that anti-IL-5 agents attenuate blood and tissue eosinophilia, the major pathophysiology of ICEP. Indeed, in the present cases, all 4 ICEP patients with frequent recurrences were well controlled by anti-IL-5 agents, and no patients have developed relapses after the treatment, as supported by previous single-case reports. ${ }^{32-36}$ Ricketti et al also reported that a patient with relapsing CEP was treated with benralizumab, and the safety of long-term treatment for over 30 months was found, with maintenance of its efficacy. ${ }^{37}$ In addition, corticosteroid treatment was withdrawn or reduced in all 4 cases, which contributed to attenuation of corticosteroid-related adverse effects (Table 3). Practically, 2 cases showed improved glucose tolerance after initiation of anti-IL-5 agents. Thus, anti-IL-5 agents might be an alternative treatment 
for ICEP patients with frequent recurrences, and expansion of the indications for anti-IL-5 agents in the treatment of ICEP would be expected. Determination of treatment selection, either mepolizumab or benralizumab, is challenging because there are few data comparing the effects of these drugs in terms of their clinical efficacy and pharmacodynamics. Moran et al reported that, for patients with eosinophilic asthma, benralizumab reduced blood eosinophils in the first 24 hours faster than mepolizumab. ${ }^{38}$ The data indicated that benralizumab has greater efficacy for blood eosinophil depletion, but the contribution to clinical outcomes for patients with ICEP is still unclear.

The present case series showed that anti-IL-5 agents had a beneficial effect for ICEP patients with frequent recurrences. To confirm the validity of the present results, multicenter, prospective, controlled studies comparing ordinary treatment to anti-IL-5 agents with a larger number of patients should be performed.

\section{Conclusion}

The present results show that relapse is frequent in ICEP patients during corticosteroid dose tapering, and this dependence on corticosteroid induces corticosteroid-related adverse events. Anti-IL-5 agents might contribute to decreasing ICEP relapses and avoiding corticosteroid-related adverse events.

\section{Funding}

This research did not receive any specific grant from funding agencies in the public, commercial, or not-for-profit sectors.

\section{Disclosure}

The authors report no conflicts of interest in this work.

\section{References}

1. Suzuki Y, Suda T. Eosinophilic pneumonia: a review of the previous literature, causes, diagnosis, and management. Allergol Int. 2019;68 (4):413-419. doi:10.1016/j.alit.2019.05.006

2. Naughton M, Fahy J, FitzGerald MX. Chronic eosinophilic pneumonia. A long-term follow-up of 12 patients. Chest. 1993;103(1):162-165. doi:10.1378/chest.103.1.162

3. Rosenberg CE, Khoury P. Approach to eosinophilia presenting with pulmonary symptoms. Chest. 2021;159(2):507-516. doi:10.1016/j. chest.2020.09.247

4. Barry J, Gadre A, Akuthota P. Hypersensitivity pneumonitis, allergic bronchopulmonary aspergillosis and other eosinophilic lung diseases. Curr Opin Immunol. 2020;66:129-135. doi:10.1016/j.coi.2020.10.005

5. Marchand E, Cordier JF. Idiopathic chronic eosinophilic pneumonia. Orphanet J Rare Dis. 2006;1:11. doi:10.1186/1750-1172-1-11

6. Suzuki Y, Suda T. Long-term management and persistent impairment of pulmonary function in chronic eosinophilic pneumonia: a review of the previous literature. Allergol Int. 2018;67(3):334-340. doi:10.1016/j.alit.2017.12.004

7. Marchand E, Reynaud-Gaubert M, Lauque D, Durieu J, Tonnel AB, Cordier JF. Idiopathic chronic eosinophilic pneumonia. A clinical and followup study of 62 cases. The Groupe d'Etudes et de Recherche sur les Maladies “Orphelines" Pulmonaires (GERM”O”P). Medicine. 1998;77 (5):299-312. doi:10.1097/00005792-199809000-00001

8. Suzuki Y, Oyama Y, Hozumi H, et al. Persistent impairment on spirometry in chronic eosinophilic pneumonia: a longitudinal observation study (Shizuoka-CEP study). Ann Allergy Asthma Immunol. 2017;119(5):422-428 e2.

9. Ishiguro T, Takayanagi N, Uozumi R, et al. The long-term clinical course of chronic eosinophilic pneumonia. Intern Med. 2016;55(17):2373-2377. doi:10.2169/internalmedicine. 55.6765

10. Oyama Y, Fujisawa T, Hashimoto D, et al. Efficacy of short-term prednisolone treatment in patients with chronic eosinophilic pneumonia. Eur Respir J. 2015;45(6):1624-1631. doi:10.1183/09031936.00199614

11. Volmer T, Effenberger T, Trautner C, Buhl R. Consequences of long-term oral corticosteroid therapy and its side-effects in severe asthma in adults: a focused review of the impact data in the literature. Eur Respir J. 2018;52(4):1800703. doi:10.1183/13993003.00703-2018

12. Ortega HG, Liu MC, Pavord ID, et al. Mepolizumab treatment in patients with severe eosinophilic asthma. N Engl J Med. 2014;371 (13):1198-1207. doi:10.1056/NEJMoa1403290

13. Bleecker ER, FitzGerald JM, Chanez P, et al. Efficacy and safety of benralizumab for patients with severe asthma uncontrolled with high-dosage inhaled corticosteroids and long-acting $\beta 2$-agonists (SIROCCO): a randomised, multicentre, placebo-controlled Phase 3 trial. Lancet. 2016;388 (10056):2115-2127. doi:10.1016/S0140-6736(16)31324-1

14. Lambrecht BN, Hammad H. The immunology of asthma. Nat Immunol. 2015;16(1):45-56.

15. Vantaggiato L, Perruzza M, Refini RM, et al. Mepolizumab and benralizumab in severe eosinophilic asthma: preliminary results of a proteomic study. Lung. 2020;198(5):761-765. doi:10.1007/s00408-020-00379-6

16. Laviolette M, Gossage DL, Gauvreau G, et al. Effects of benralizumab on airway eosinophils in asthmatic patients with sputum eosinophilia. J Allergy Clin Immunol. 2013;132(5):1086-1096 e5. doi:10.1016/j.jaci.2013.05.020

17. Kolbeck R, Kozhich A, Koike M, et al. MEDI-563, a humanized anti-IL-5 receptor alpha mAb with enhanced antibody-dependent cell-mediated cytotoxicity function. $J$ Allergy Clin Immunol. 2010;125(6):1344-1353 e2. doi:10.1016/j.jaci.2010.04.004 
18. Menzies-Gow A, Flood-Page P, Sehmi R, et al. Anti-IL-5 (mepolizumab) therapy induces bone marrow eosinophil maturational arrest and decreases eosinophil progenitors in the bronchial mucosa of atopic asthmatics. J Allergy Clin Immunol. 2003;111(4):714-719. doi:10.1067/ mai.2003.1382

19. Marchand E, Etienne-Mastroianni B, Chanez P, et al. Idiopathic chronic eosinophilic pneumonia and asthma: how do they influence each other? Eur Respir J. 2003;22(1):8-13.

20. Durieu J, Wallaert B, Tonnel AB. Long-term follow-up of pulmonary function in chronic eosinophilic pneumonia. Groupe d'Etude en Pathologie Interstitielle de la Societe de Pathologie Thoracique du Nord. Eur Respir J. 1997;10(2):286-291.

21. Matsuse H, Shimoda T, Fukushima C, et al. Diagnostic problems in chronic eosinophilic pneumonia. J Int Med Res. 1997;25(4):196-201. doi:10.1177/030006059702500404

22. Kubo H, Loegering DA, Adolphson CR, Gleich GJ. Cytotoxic properties of eosinophil granule major basic protein for tumor cells. Int Arch Allergy Immunol. 1999;118(2-4):426-428. doi:10.1159/000024154

23. McBrien CN, Menzies-Gow A. The biology of eosinophils and their role in asthma. Front Med. $2017 ; 4: 93$.

24. Fukuchi M, Miyabe Y, Furutani C, et al. How to detect eosinophil ETosis (EETosis) and extracellular traps. Allergol Int. 2021;70(1):19-29. doi:10.1016/j.alit.2020.10.002

25. Adachi T, Alam R. The mechanism of IL-5 signal transduction. Am J Physiol. 1998;275(3):C623-C633.

26. Larose MC, Turcotte C, Chouinard F, et al. Mechanisms of human eosinophil migration induced by the combination of IL-5 and the endocannabinoid 2-arachidonoyl-glycerol. J Allergy Clin Immunol. 2014;133(5):1480-1482, 2 e1-3. doi:10.1016/j.jaci.2013.12.1081

27. Mould AW, Ramsay AJ, Matthaei KI, Young IG, Rothenberg ME, Foster PS. The effect of IL-5 and eotaxin expression in the lung on eosinophil trafficking and degranulation and the induction of bronchial hyperreactivity. J Immunol. 2000;164(4):2142-2150. doi:10.4049/jimmunol.164.4.2142

28. Katoh S, Matsumoto N, Matsumoto K, Fukushima K, Matsukura S. Elevated interleukin-18 levels in bronchoalveolar lavage fluid of patients with eosinophilic pneumonia. Allergy. 2004;59(8):850-856. doi:10.1111/j.1398-9995.2004.00492.x

29. Miyazaki E, Nureki S, Fukami T, et al. Elevated levels of thymus- and activation-regulated chemokine in bronchoalveolar lavage fluid from patients with eosinophilic pneumonia. Am J Respir Crit Care Med. 2002;165(8):1125-1131. doi:10.1164/ajrccm.165.8.2106110

30. Castro M, Zangrilli J, Wechsler ME, et al. Reslizumab for inadequately controlled asthma with elevated blood eosinophil counts: results from two multicentre, parallel, double-blind, randomised, placebo-controlled, phase 3 trials. Lancet Respir Med. 2015;3(5):355-366. doi:10.1016/S22132600(15)00042-9

31. Kelly EA, Esnault S, Liu LY, et al. Mepolizumab attenuates airway eosinophil numbers, but not their functional phenotype, in asthma. Am J Respir Crit Care Med. 2017;196(11):1385-1395. doi:10.1164/rccm.201611-2234OC

32. Takano T, Inutsuka Y, Nakamura S, Ando N, Komori M. Benralizumab use in chronic eosinophilic pneumonia with eosinophilic bronchiolitis and chronic airway infection. Respirol Case Rep. 2021;9(8):e00810. doi:10.1002/rcr2.810

33. Kisling A, Jones J, Hixson C, Hostler D, Hostler J. Mepolizumab: an alternative therapy for idiopathic chronic eosinophilic pneumonia with glucocorticoid intolerance. Drugs Context. 2020;9:1-7. doi:10.7573/dic.2020-5-3

34. Izumo T, Kuse N, Awano N, et al. Rapid and sustained effects of a single dose of benralizumab on chronic eosinophilic pneumonia. Respir Med Case Rep. 2020;30:101062.

35. Shimizu Y, Kurosawa M, Sutoh Y, Sutoh E. Long-term treatment with anti-interleukin 5 antibodies in a patient with chronic eosinophilic pneumonia. J Investig Allergol Clin Immunol. 2020;30(2):154-155. doi:10.18176/jiaci.0468

36. To M, Kono Y, Yamawaki S, et al. A case of chronic eosinophilic pneumonia successfully treated with mepolizumab. J Allergy Clin Immunol Pract. 2018;6(5):1746-1748 e1. doi:10.1016/j.jaip.2018.06.017

37. Ricketti PA, Ricketti AJ. Relapsing chronic eosinophilic pneumonia treated successfully with benralizumab. Ann Allergy Asthma Immunol. 2021;126(6):726-727. doi:10.1016/j.anai.2021.02.016

38. Moran AM, Ramakrishnan S, Borg CA, et al. Blood eosinophil depletion with mepolizumab, benralizumab, and prednisolone in eosinophilic asthma. Am J Respir Crit Care Med. 2020;202(9):1314-1316. doi:10.1164/rccm.202003-0729LE

Journal of Asthma and Allergy

Dovepress

\section{Publish your work in this journal}

The Journal of Asthma and Allergy is an international, peer-reviewed open-access journal publishing original research, reports, editorials and commentaries on the following topics: Asthma; Pulmonary physiology; Asthma related clinical health; Clinical immunology and the immunological basis of disease; Pharmacological interventions and new therapies. The manuscript management system is completely online and includes a very quick and fair peer-review system, which is all easy to use. Visit http://www.dovepress.com/testimonials.php to read real quotes from published authors.

Submit your manuscript here: https://www.dovepress.com/journal-of-asthma-and-allergy-journal 